\title{
Highly parallel gene-to-BAC addressing using microarrays
}

Hui Liu ${ }^{1}$, Jim McNicol ${ }^{2}$, Micha Bayer ${ }^{1}$, Jenny A. Morris ${ }^{1}$, Linda Cardle ${ }^{1}$, David F. Marshall ${ }^{1}$, Daniela Schulte ${ }^{3}$, Nils Stein ${ }^{3}$, Bu-Jun Shi ${ }^{4}$, Stefan Taudien ${ }^{5}$, Robbie Waugh ${ }^{1}$, and Peter E. Hedley ${ }^{1}$ ${ }^{1}$ Programme of Genetics, Scottish Crop Research Institute, Dundee, UK, ${ }^{2}$ Biomathematics and Statistics Scotland, Dundee, UK, ${ }^{3}$ Leibniz Institute of Plant Genetics and Crop Plant Research, Gatersleben, Germany, ${ }^{4}$ Australian Centre for Plant Functional Genomics, University of Adelaide, Glen Osmond, Australia, and ${ }^{5}$ Leibniz Institute for Age Research, Fritz Lipmann Institute (FLI), Jena, Germany

BioTechniques 50:165-174 (March 2011) doi 10.2144/000113627

Keywords: Physical maps; genetic maps; microarrays; anchoring

Supplementary material for this article is available at www.BioTechniques.com/article/113627.

Second-generation sequencing now provides the potential for low-cost generation of whole-genome sequences. However, for large-genome organisms with high repetitive DNA content, genome-wide short read sequence assembly is currently impossible, with accurate ordering and localization of genes still relying heavily on integration with physical and genetic maps. To facilitate this process, we have used Agilent microarrays to simultaneously address thousands of gene sequences to individual BAC clones and contiguous sequences that form part of an emerging physical map of the large and currently unsequenced 5.3-Gb barley genome. The approach represents a cost-effective, highly parallel alternative to traditional addressing methods. By coupling the gene-to-BAC address data with gene-based molecular markers, thousands of BACs can be anchored directly to the genetic map, thereby generating a framework for orientating and ordering genes, and providing direct links to phenotypic traits.

Physical map-based genome sequencing strategies have recently been superseded by relatively low-cost but 'quick and dirty' second-generation sequencing (2GS) based on whole-genome shotgun approaches $(1,2)$. However, for genomes that comprise a high proportion of repetitive DNA and gene families that have evolved by duplication, de novo assembly of 2 GS data remains problematic, at best providing information restricted to low copy regions (3). While this type of output has considerable and immediate utility, it remains intrinsically limited through a lack of context, encompassing little information about gene location or order. Current 2GS draft assemblies of large and complex genomes are therefore a temporary substitute for a gold standard reference sequence which remains the primary goal of most research communities.

Based on current technology, such a reference sequence can still realistically only be delivered by robust but expensive physical map-based genome sequencing (4). This is typically centered on high information content fingerprinting (HICF) of bacterial artificial chromosome (BAC) clones that are assembled into contiguous sequences (contigs) prior to sequencing the Minimal
Tiling Path (MTP) of clones $(5,6)$. The physical map overcomes issues associated with large recombinationally inert regions typical of large and repetitive genomes, and provides the necessary context for exploring genome structure and organization, both within and among species. A key step in the physical map-based sequencing strategy is anchoring individual BAC clones and contigs to high-resolution genetic maps. This provides both a framework for orientating and ordering contigs, as well as a direct connection to mapped phenotypic traits (4). However, currently established anchoring techniques tend to be low-throughput or involve many processing steps (7).

We describe a novel application of DNA microarray technology to potentially anchor thousands of genes to thousands of BACs. The work was conducted on the large [ 5.3 $\mathrm{Gb}(8)$ ] unsequenced barley genome but is generally applicable to any organism for which $\mathrm{BAC}$ resources and genetic maps exist.

\section{Materials and methods}

Barley BAC library pooling strategy

Genomic DNA from barley cultivar Morex was used to construct a BAC library (HVVMRXALLeA) in pIndigoBac536.
The original library contained 147,840 BAC clones (in 385384 -well plates) with an average insert size of $125 \mathrm{~kb}$, giving a total of $\sim 3.7 \times$ barley genome coverage. The BAC library was divided by the commercial vendor Amplicon Express (Pullman, WA, USA) into 55 sequential Super Pools (SPs), each consisting of seven 384-well plates. For each SP, BAC DNA was initially pooled into seven sets corresponding to each of the 384-well plates, 16 sets corresponding to each of the 384-well plate rows of all seven plates, and 24 sets corresponding to each of the 384-well plate columns of all seven plates. The seven plate, 16 row, and 24 column sets were then further pooled to create five Matrix Plate Pools (MPPs), eight Matrix Row Pools (MRPs), and 10 Matrix Column Pools (MCPs), respectively, for a total of 23 Matrix Pools (MPs). This Matrix pooling strategy results in each $\mathrm{BAC}$ being contained in two different MPs for each type of MP (Plate, Row, or Column) (i.e., in two of the five MPPs, two of the eight MRPs, and two of the 10 MCPs). The strategy for pooling, hybridization and deconvolution of the BACs is summarized in Figure 1.

Amplification of pooled BAC DNA SP and MP BAC DNAs were supplied as above. As the concentration of pooled 


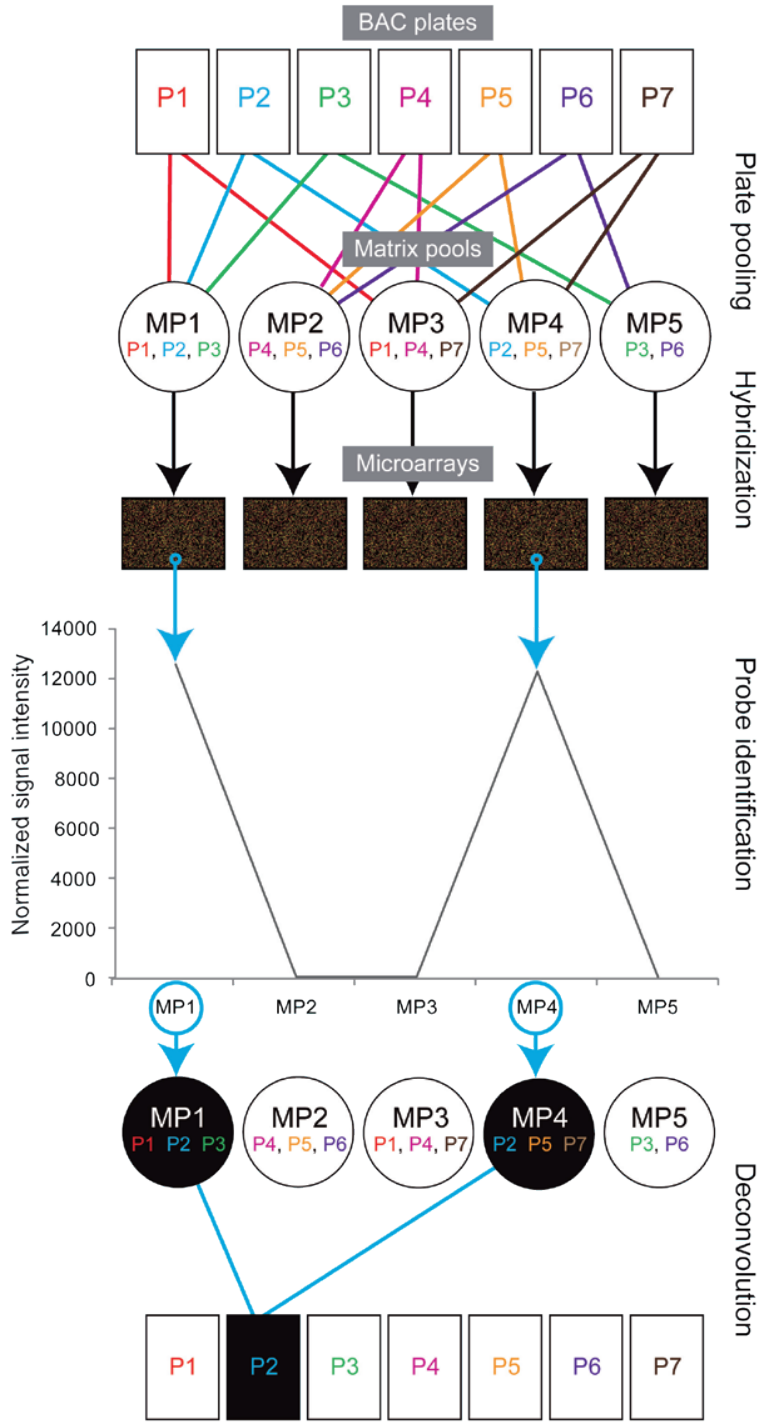

Figure 1. Schematic of BAC pooling, microarray hybridization, and deconvolution procedure. Only the strategy for processing Matrix Plate Pools (which are labelled and referred to simply as Matrix Pools in this figure) is shown, but the same principle applies to the Matrix Row and Column pools. BAC clones, each from a single plate, are initially pooled together (P1-P7), followed by a second pooling to form the Matrix Pools (MP1-MP5), such that each BAC appears in two independent MPs. Following hybridization of amplified MP DNA to microarrays, probes are identified with significant signal in two of the MPs (for single-copy genes). Deconvolution enables identification of the original plate (in this example, P2) containing a BAC clone with a gene fragment corresponding to the microarray probe sequence. This is combined with the row and column deconvolution data to determine the full original BAC addresses.

DNA supplied was very low $(\sim 5 \mathrm{ng} / \mu \mathrm{L})$, high-concentration stocks were produced from each pool using the phi29 DNA polymerase rolling circle amplification method (GenomiPhi V2 DNA Polymerase Kit, GE Healthcare, Piscataway, NJ, USA). Amplification conditions were as recommended by the manufacturer: $2 \mu \mathrm{L}$ each DNA pool was denatured in $9 \mu \mathrm{L}$ sample buffer at $95^{\circ} \mathrm{C}$ for $5 \mathrm{~min}$ and subsequently placed on ice. A mixture of $1 \mu \mathrm{L}$ GenomiPhi phi29 DNA polymerase and $9 \mu \mathrm{L}$ reaction buffer, containing dNTPs and random hexamers, were added to the denatured samples. After $2 \mathrm{~h}$ at $30^{\circ} \mathrm{C}$, the reaction was terminated by heating for $10 \mathrm{~min}$ at $65^{\circ} \mathrm{C}$. DNA yield and quality were checked on $0.7 \%$ agarose.

Microarray labeling and hybridization A custom microarray SCRI Hv35 44k v1 was designed (design 020599; Agilent, Santa Clara, CA, USA) representing 42,302 barley expressed sequence tag (EST) contig sequences. Sequences for this design were selected from a total of 50,938 putative unigenes from HarvEST assembly 35 (www.harvest-web.org) representing $\sim 450,000$ ESTs. Selection was based upon contig orientation derived from (i) homology to the nonredundant protein database (NCBI nr), (ii) homology to ESTs from directional cDNA libraries, and (iii) presence of a polyA tract. The microarray was designed with one 60 -mer probe per selected unigene in $4 \times 44 \mathrm{k}$ format using default parameters in the Agilent eArray software (https://earray.chem.agilent.com/ earray). All of the probes were designed to be within or close to the $3^{\prime}$ untranslated regions of the ESTs to maximize selection of unique probe sequences. In addition, the entire EST collection was utilized for cross reference within eArray software to minimize chances of nonspecific hybridization. Full details can be found at ArrayExpress (www.ebi.ac.uk/arrayexpress; accession no. A-MEXP-1728)

Two-channel processing of the microarrays was used, with the entire set of MP DNAs from one SP labeled entirely with $\mathrm{Cy} 3$ and the entire set of MP DNAs from another SP labeled with Cy5. In total, 23 arrays were utilized for the two complete sets of MPs (equivalent to two SPs) described in the text. Briefly, amplified MP DNA (200 ng) was labeled using a modified BioPrime Array CGH Genomic DNA Labeling System (Invitrogen, Carlsbad, CA, USA): amplified MP DNA in $11 \mu \mathrm{L}$ was added to $10 \mu \mathrm{L}$ $2.5 \times$ random primer reaction buffer mix and denatured at $95^{\circ} \mathrm{C}$ for 5 min prior to cooling on ice. To this was added $2.5 \mu \mathrm{L}$ modified $10 \times$ dNTPs buffer $1.2 \mathrm{mM}$ each of dATP, dGTP, dTTP; $0.6 \mathrm{mM} \mathrm{dCTP}$; $10 \mathrm{mM}$ Tris pH8.0; $1 \mathrm{mM}$ EDTA), $1 \mu \mathrm{L}$ either Cy 3 or Cy $5 \mathrm{dCTP}(1 \mathrm{nM})$ and $0.5 \mu \mathrm{L}$ Klenow enzyme (20 U) and incubated for $16 \mathrm{~h}$ at $37^{\circ} \mathrm{C}$. Labeled samples for each array were combined and unincorporated dyes removed using Qiaquick PCR Purification Kit (Qiagen, Valencia, CA, USA) as recommended, eluting with $20 \mu \mathrm{L}$ EB buffer (Qiagen). Hybridizations and washing were performed as recommended (Agilent Protocol v5.5). Scanning was performed with an Agilent G2505B scanner using default settings and data extracted using Agilent FE software (v9.5.3). The experimental design and all microarray data have been submitted to ArrayExpress (www.ebi.ac.uk/arrayexpress; accession no. E-TABM-1017).

Data preprocessing and deconvolution Data were preprocessed and normalized using GeneSpring software (v7.3; Agilent). Extracted data from each of the two sets of MP arrays were imported separately and normalization performed as for singlechannel arrays (data transformation 
of individual probe measurements to a minimum of 0.01 ; per chip normalization to the 50 th percentile). Data were exported from GeneSpring for downstream analysis.

Deconvolution of plate, row, and column pools was performed using scripting (Supplementary Material) in GenStat (VSN International, Hemel Hempstead, UK) by identifying maximum signal above background in two out of five MPPs, two out of eight MRPs, and two out of 10 MCPs (see strategy in Figure 1). Identification of a unigene in two MPPs, for example, was measured by the log ratio of the difference between the second- and third-largest MPP signals and the difference between the third largest and the median probe signal of the remaining MPPs. As we are expecting two significant outlying signals from each MP type (for a single copy gene), this approach identifies those pools with the first- and secondhighest significant probe signals above background. This ratio was then weighted using a Gompertz function and identification confirmed when the weighted ratio (gScore) exceeded an empirically assigned threshold (0.9). A match with the BAC clone was confirmed when the combined identified unigene plates, rows and columns corresponded to the known BAC pooling addresses.

\section{Illumina sequencing of SP1 DNA}

Separate samples (five in total) of amplified SP1 DNA were sequenced on an Illumina GAII sequencer (San Diego, CA, USA), with a single lane used for each sample. This resulted in a total of $9,269,872$ reads of 54-nucleotide length and 57,650,261 reads of 70 -nucleotide length $(4.5 \mathrm{~Gb}$ total). The sequence reads were quality-

A

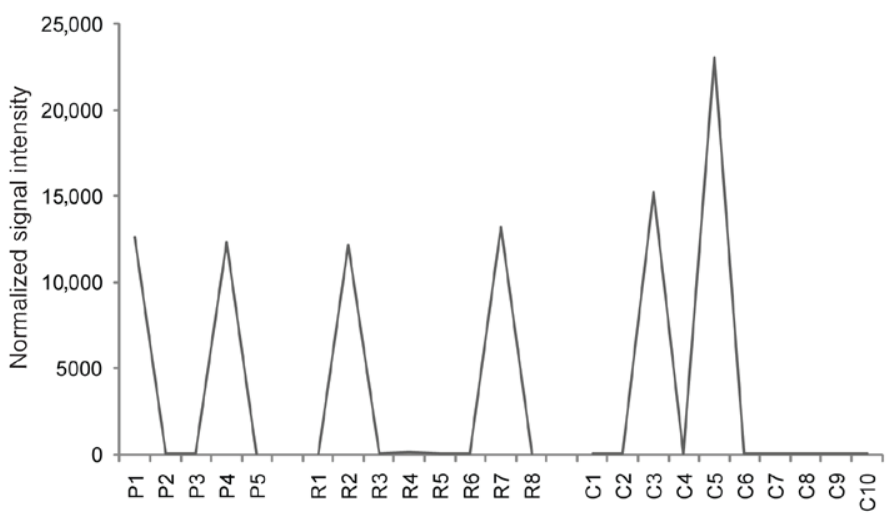

B
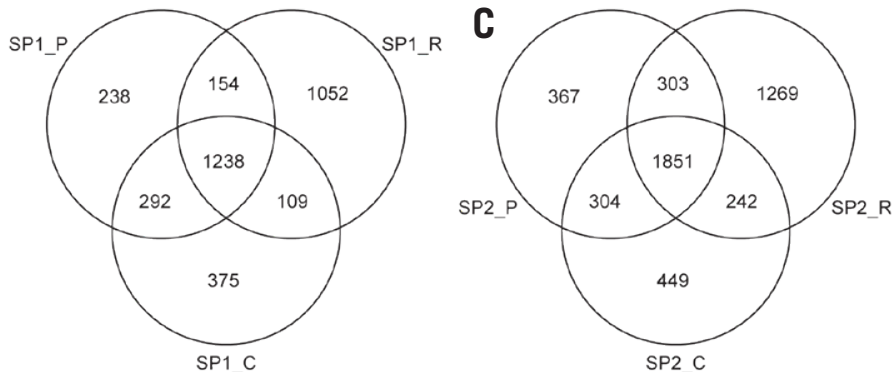

Figure 2. Microarray signal detection and deconvolution. (A) Example of signal patterns from plate (P1-P5), row (R1-R8), and column (C1-C10) matrix pools ( $y$ axis indicates normalized signal intensity). Positive signals in this example are from P1, P4, R2, R7, C3, and C5. (B and C) Venn diagram of overlaps between single unigenes from deconvoluted single-hit BACs of SP1 (B) and SP2 (C).

trimmed with a quality score cutoff of 20 to remove poor-quality sequence at the start and end of reads. This left a total of $66,827,380$ reads of average length of 62 nucleotides. The combined trimmed reads from all samples were mapped to the Harvest 35 unigene reference sequences using both MOSAIK and SOAPAligner (9) read-mapping tools, in order to be able to compare different outcomes and to eliminate tool-based bias. For each of the mapping programs, two mismatches were allowed.
Validation using quantitative PCR Assays were designed using the online software of the Universal Probe Library (www.roche-applied-science.com/sis/ rtpcr/upl/index.jsp; Roche, Basel, Switzerland) (Supplementary Table S4). Quantitative PCR reactions of BAC-pooled DNA with SYBR Green I were performed on 7500 Fast equipment (Applied Biosystems, Foster City, CA, USA). A total reaction volume of $10 \mu \mathrm{L}$ included $2 \mu \mathrm{L}$ unamplified BAC pool DNA (10 ng), $5 \mu \mathrm{L}$ SYBR Green I PCR Master mix (Sigma-

Table 1. Summary of microarray-based BAC pool screening.

\begin{tabular}{|c|c|c|c|c|c|c|c|c|c|c|}
\hline \multirow{2}{*}{$\begin{array}{c}\text { Super } \\
\text { pool }\end{array}$} & \multirow{2}{*}{$\begin{array}{c}\text { Unigenes } \\
\text { (single) }\end{array}$} & \multirow{2}{*}{$\begin{array}{l}\text { Unigenes } \\
\text { (double) }\end{array}$} & \multirow{2}{*}{$\begin{array}{l}\text { Unigenes } \\
\text { (total) }\end{array}$} & \multirow{2}{*}{$\begin{array}{l}\text { Unique } \\
\text { BACs } \\
\text { (single) }\end{array}$} & \multirow{2}{*}{$\begin{array}{l}\text { Unique } \\
\text { BACs } \\
\text { (double) }\end{array}$} & \multirow{2}{*}{$\begin{array}{c}\text { Unique } \\
\text { BACs } \\
\text { (total) }\end{array}$} & \multicolumn{4}{|c|}{ Genetic mapping annotation } \\
\hline & & & & & & & BOPA & Agilent eQTL & Affy eQTL & Unique map positions \\
\hline SP1 & 1238 & 33 & 1271 & 531 & 64 & 595 & 82 & 123 & 227 & 155 \\
\hline SP2 & 1851 & 15 & 1866 & 833 & 26 & 859 & 141 & 176 & 363 & 197 \\
\hline Total & 3089 & 48 & 3137 & 1364 & 90 & 1454 & 223 & 299 & 590 & 352 \\
\hline \multicolumn{11}{|c|}{$\begin{array}{l}\text { Numbers of probes (unigenes) and single/double hit BACs identified by microarray addressing and their associated genetic map locations derived from Illumina } \\
\text { Goldengate- (BOPA) and eQTL- (Agilent' } \text { and Affymetrixc) based mapping. } \\
\text { aClose, T.J., P.R. Bhat, S. Lonardi, Y. Wu, N. Rostoks, L. Ramsay, A. Druka, N. Stein, et al. 2009. Development and implementation of high-throughput SNP genotyping } \\
\text { in barley. BMC Genomics 10:582. } \\
\text { 'Chen, X., C.A. Hackett, R.E. Niks, P.E. Hedley, C. Booth, A. Druka, T.C. Marcel, A. Vels, et al. 2010. An eQTL analysis of partial resistance to Puccinia hordei in barley. } \\
\text { PLoS One 5:e8598. } \\
\text { 'Potokina, E., A. Druka, Z. Luo, R. Wise, R. Waugh, and M. Kearsey. 2008. Gene expression quantitative trait locus analysis of } 16000 \text { barley genes reveals a complex } \\
\text { pattern of genome-wide transcriptional regulation. Plant J. 53:90-101. }\end{array}$} \\
\hline
\end{tabular}




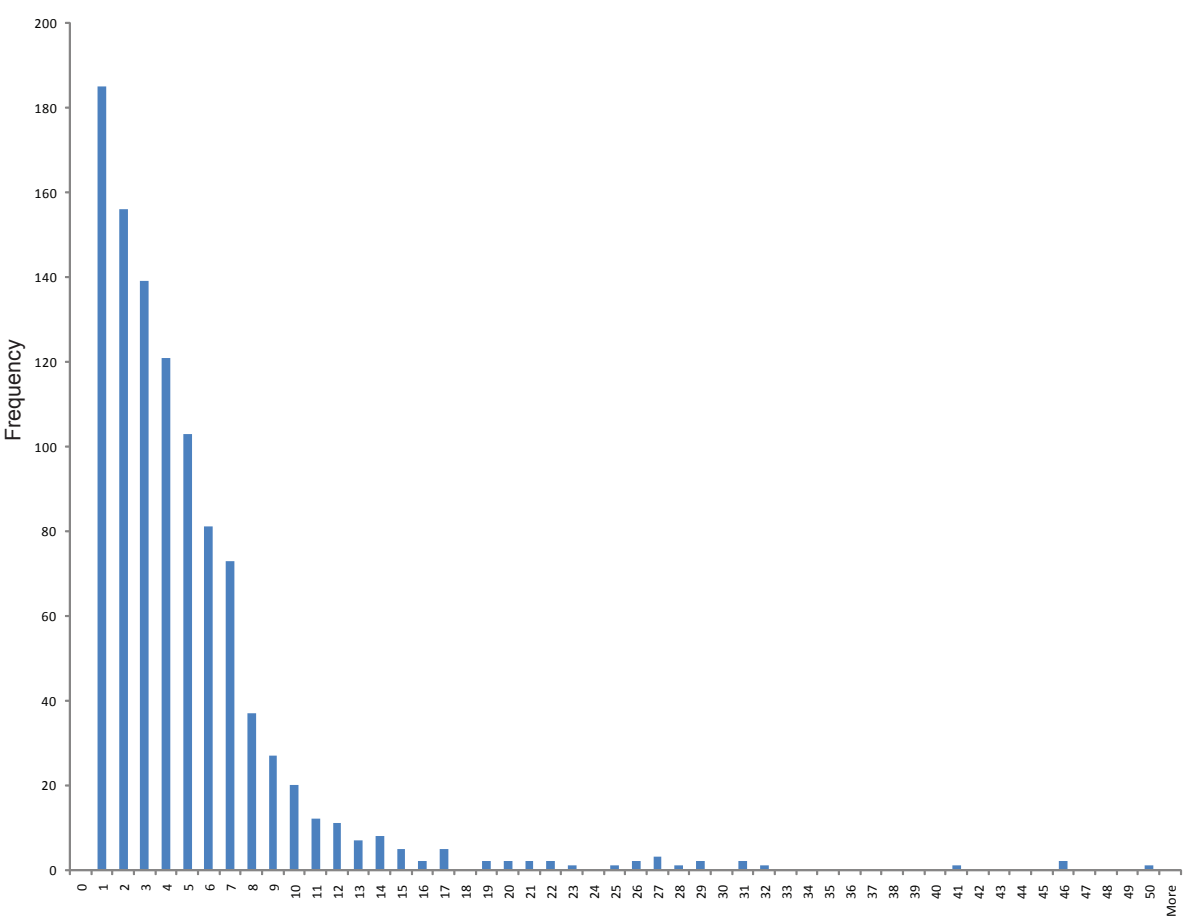

Bin

Figure 3. Histogram of frequencies (bin $=1$ ) of nucleotide coverage of mapped 2GS Illumina reads to microarray-positive unigenes. DNA from a single amplified SuperPool (SP1) was subjected to Illumina sequencing, resulting in over 9 million reads. A pronounced bias toward low representation of mapped reads can be seen, with an average coverage of only $4.4 \times$. However, $~ 87 \%$ of microarraypositive unigenes were validated by sequencing.

Aldrich, Dorset, UK) and gene-specific primers $(500 \mathrm{nM}$ each). The PCR program was performed as follows: 1 cycle of $95^{\circ} \mathrm{C}$ for $10 \mathrm{~min}$, followed by 40 cycles of $95^{\circ} \mathrm{C}$ for $15 \mathrm{~s}$ and $60^{\circ} \mathrm{C}$ for $60 \mathrm{~s}$. Following amplification, a reaction product melting curve analysis was performed $\left(95^{\circ} \mathrm{C}\right.$ for $15 \mathrm{~s}, 58^{\circ} \mathrm{C}$ for $1 \mathrm{~min}$ and $95^{\circ} \mathrm{C}$ for $15 \mathrm{~s}$ ) to provide evidence for a single reaction product.

\section{Results and discussion}

We designed and used an Agilent custom barley array (ArrayExpress: A-MEXP1728), representing 42,302 expressed genes, to screen whole genome-amplified DNA derived from multidimensional pools of BAC clones that were also incorporated into the HICF physical map (data not shown). The barley BAC library consists of 147,840 clones which were sequentially assigned to 55 Super Pools (SPs) with seven 384-well plates per SP. Plate, row, and column pools from each SP were further pooled, respectively, into five Matrix Plate Pools (MPPs), eight Matrix Row Pools (MRPs), and 10 Matrix Column Pools (MCPs), for a total of 23 Matrix Pools (MPs) (see "Barley BAC library pooling strategy" in the Materials and methods). Each SP is estimated to represent $\sim 1 / 16$ th of the whole barley genome, a feature that reduces the problem of multiple addressing when deconvoluting gene to $\mathrm{BAC}$ associations. To date, we have screened a $1 \times$ equivalent of the barley genome (i.e., the MPs from 16 SPs; data not shown) to couple geneto-BAC addresses as a contribution to the International Barley Genome Sequencing Consortium effort (10). We describe here the proof-of-principle involving two SPs: SP1 and SP2.

We independently labeled each MP of SP1 with Cy3 and each MP of SP2 with Cy5, and then co-hybridized each of the 23 pairs of labeled MPs (one MP from each SP) to one of our custom barley microarrays. Therefore, 23 microarrays in total were used to screen all the MPs of both SPs. Following signal extraction from scanned images, data were imported into GeneSpring software (Agilent) to normalize overall signal between each MP microarray. Downstream data processing scripts were written in GenStat software (VSN International) (Supplementary Material) to create a two-stage semi-automated procedure for the identification of $\mathrm{BAC}$ clones containing genes corresponding to probes on the microarray. Both stages exploit features of the MP strategy. The first identifies, separately for the plate, row, and column MPs, those probes which have high signals only two times in a single dimension (Figure $2 \mathrm{~A})$. The second combines this information across dimensions (plates, rows, and columns) and matches the output with the known BAC-pooling patterns, to allow deconvolution and identification of the individual $\mathrm{BAC}$ clones that contain genes corresponding to probes on the microarray (Figure 2, B and C). The scripts can simultaneously deconvolute single and double $\mathrm{BAC}$ hits (the latter have two addresses in one of the three dimensions due to high signals in three MPs for that dimension), with the following criteria: ( $i$ ) the minimum gScore (weighted log-ratio) of plate, row, and column MPs must be $>0.9$ for both single and double BAC hits; (ii) for a single $\mathrm{BAC}$ hit, the third-highest signal in plate, row, and column MPs must be $<100$; for double BAC hits, the fourth highest signal in plate, row, and column MPs must be $<100$. If any of these criteria was not met, the probe was filtered out. After filtering, a total of 1364 BACs (531 from SP1, 833 from SP2) hybridized to 3089 microarray probes (Table 1). This included 738 BACs that hybridized to two or more probes and 626 BACs with a unique hit. Of 74 probes that had double BAC hits, 26 were adjacent in the stock plates, suggesting contamination during processing, and were therefore removed. From the annotation of the positive probes, genetic map locations were available for $25 \%$ of the corresponding unigenes, providing genetic anchoring information for 352 BACs from SP1 and SP2 (Table 1; Supplementary Table S1).

In order to validate the microarray hybridization results, four approaches were taken. Firstly, we exploited the strong syntenic relationship between barley and the fully sequenced model cereal Brachypodium (11). We examined the 606 of the 738 BACs that hybridized to two or more probes that also had significant homology to Brachypodium genes. For 172 of these BACs, multiple probes matched single Brachypodium genes reflecting known redundancy on the microarray, thereby providing independent cross validation of the microarray hybridization results. For $434 \mathrm{BACs}$, the barley probes matched multiple Brachypodium genes. Consistent with expected levels of conservation of synteny among grass genomes, 200 (46.1\%) BACs hybridized to probes matching adjacent Brachypodium genes, thereby providing further validation, and 234 (53.9\%) matched nonadjacent genes (Supplementary Table S2).

We then looked for the corresponding barley unigenes in 28 positively hybridizing BAC clones from SP1 that are both present in the emerging barley HICF 


\section{Acknowledgments}

The research leading to these results has received funding from the European Community's Seventh Framework Programme (FP7/ 2007-2013) under grant agreement FP7-212019. Access to the partial physical map and associated sequences of barley was kindly provided by Matthias Platzer of Leibniz Institute for Age Research, Fritz Lipmann Institute, Jena, Germany, and Burkhard Steuernagel and Uwe Scholz of Leibniz Institute of Plant Genetics and Crop Plant Research, Gatersleben, Germany.

\section{Competing interests}

The authors declare no competing interests.

\section{References}

1. Gonzalez, V.M., J. Garcia-Mas, P. Arus, and P. Puigdomenech. 2010. Generation of a BAC-based physical map of the melon genome. BMC Genomics 11:339.

2.Li, R., W. Fan, G. Tian, H. Zhu, L. He, J. Cai, Q. Huang, Q. Cai, et al. 2010. The sequence and de novo assembly of the giant panda genome. Nature 463:311-317.
3. Schatz, M.C., A.L. Delcher, and S.L. Salzberg. 2010. Assembly of large genomes using second-generation sequencing. Genome Res. 20:1165-1173.

4.Paux, E., P. Sourdille, J. Salse, C. Saintenac, F. Choulet, P. Leroy, A. Korol, M. Michalak, et al. 2008. A physical map of the 1-gigabase bread wheat chromosome 3B. Science 322:101-104.

5. Brenner, S. and K.J. Livak. 1989. DNA fingerprinting by sampled sequencing. Proc. Natl. Acad. Sci. USA 86:8902-8906.

6.Ding, Y., M.D. Johnson, R. Colayco, Y.J. Chen, J. Melnyk, H. Schmitt, and H. Shizuya. 1999. Contig assembly of bacterial artificial chromosome clones through multiplexed fluorescence-labeled fingerprinting. Genomics 56:237-246.

7. Luo, M.C., K. Xu, Y. Ma, K.R. Deal, C.M. Nicolet, and J. Dvorak. 2009. A highthroughput strategy for screening of bacterial artificial chromosome libraries and anchoring of clones on a genetic map constructed with single nucleotide polymorphisms. BMC Genomics 10:28.

8. Bennett, M.D. and J.B. Smith. 1976 Nuclear DNA amounts in angiosperms. Philos. Trans. R. Soc. Lond. B Biol. Sci. 274:227274.

9. Li, R., C. Yu, Y. Li, T.W. Lam, S.M. Yiu, K. Kristiansen, and J. Wang. 2009. SOAP2: an improved ultrafast tool for short read alignment. Bioinformatics 25:1966-1967.
10. Schulte, D., T.J. Close, A. Graner, P. Langridge, T. Matsumoto, G. Muehlbauer, K. Sato, A.H. Schulman, et al. 2009. The international barley sequencing consortium-at the threshold of efficient access to the barley genome. Plant Physiol. 149:142-147.

11. The International Brachypodium Initiative. 2010. Genome sequencing and analysis of the model grass Brachypodium distachyon. Nature 463:763-768.

12.Hughes, T.R., M. Mao, A.R. Jones, J. Burchard, M.J. Marton, K.W. Shannon, S.M. Lefkowitz, M. Ziman, et al. 2001. Expression profiling using microarrays fabricated by an ink-jet oligonucleotide synthesizer. Nat. Biotechnol. 19:342-347.

Received 29 October 2010; accepted 25 January 2011

Address correspondence to Peter Hedley, Programme of Genetics, Scottish Crop Research Institute, Dundee, UK, e-mail: pete.hedley@ scri.ac.uk, or Robbie Waugh, Programme of Genetics, Scottish Crop Research Institute, Dundee, UK, e-mail: robbie.waugh@scri.ac.uk

To purchase reprints of this article, contact: carmelitag@fosterprinting.com

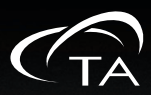

Powerful new calorimeters for the life scientist PROTEIN BINDING \& PROTEIN STABILITY 